\title{
Illumination Distribution from Shadows
}

\author{
Imari Sato Yoichi Sato Katsushi Ikeuchi \\ Institute of Industrial Science, The University of Tokyo \\ 7-22-1 Roppongi, Minato-ku, Tokyo 106-8558, Japan \\ \{imarik, ysato, ki\}@iis.u-tokyo.ac.jp
}

\begin{abstract}
The image irradiance of a three-dimensional object is known to be the function of three components: the distribution of light sources, the shape, and reflectance of a real object surface. In the past, recovering the shape and reflectance of an object surface from the recorded image brightness has been intensively investigated. On the other hand, there has been little progress in recovering illumination from the knowledge of the shape and reflectance of a real object. In this paper, we propose a new method for estimating the illumination distribution of a real scene from image brightness observed on a real object surface in that scene. More specifically, we recover the illumination distribution of the scene from a radiance distribution inside shadows cast by an object of known shape onto another object surface of known shape and reflectance. By using the occlusion information of the incoming light, we are able to reliably estimate the illumination distribution of a real scene, even in a complex illumination environment.
\end{abstract}

\section{Introduction}

The image irradiance of a three-dimensional object is known to be the function of the following three components: the distribution of light sources, the shape, and reflectance of a real object surface. From the relationship among them, three kinds of analyses on the recorded image brightness are derived: recovering the surface shape from the surface reflectance and illumination of the scene, recovering the surface reflectance from the surface shape and illumination of the scene, and recovering illumination from the shape and the reflectance of the surface.

In the past, the first two kinds of analyses, the shape recovery and the reflectance recovery, have been intensively studied using the shape from shading method $[7,8,9,16]$ as well as through reflectance analysis research $[1,12,13$, $15,18]$. In contrast, there has been little progress on the subject of recovering illumination from the knowledge of the shape and the reflectance of an object surface. This is because real scenes usually include both direct and indirect illumination distributed in a complex way and it is difficult to obtain correct illumination models to be used for the estimation. Most of the previously proposed methods related with the first two kinds of analyses aimed to estimate illuminant direction and color in a very specific illumination condition such as a case where there would be only one direct light source in the scene. Accordingly, those methods cannot be applied to the images taken under natural illumination environments.

The purpose of this study is to present our progress in recovering an illumination distribution of a real scene from the knowledge of the shape and reflectance of an real object. In the proposed method, we use radiance changes inside shadows rather than appearance changes on the surface due to the 3D geometry of the surface and the illuminant direction. More specifically, we estimate an illumination distribution of the scene by observing a radiance distribution inside shadows cast by an object of known shape onto another object surface of known shape and reflectance. Shadows in a real scene are caused by the occlusion of incoming lights as illustrated in Figure 1, and thus shadows contain various pieces of information about the illumination of the scene. Nevertheless, in the past, shadows have been used for determining the 3D shapes and orientations of an object which cast shadows onto the scene $[2,11,14,19]$, while very few studies have focused on the the illuminant information which shadows could provide.

In the proposed method, we are able to reliably estimate an illumination distribution of a real scene by making use of the occlusion information of the incoming light. Also, our method is applicable to the images taken under a complex illumination environment such as images taken in an ordinary room, including reflections from the wall and other objects in the scene.

\subsection{Overview}

Before we describe the proposed method in detail, we should clarify the basic steps of our method.

First, we take an image of the scene using a color CCD camera so that shadows of an object appear in the image. In the rest of the paper, we refer to the image with shadows as the shadow image and the object of known shape, which cast shadows onto the scene, as the occluding object. (A typical example of a shadow image is shown in Figure 3.)

Then, based on the radiance distribution inside shadows, 
an illumination distribution of a real scene is estimated as a collection of imaginary point light sources distributed over the entire scene. The key idea of the proposed method is the discretization of the overall illumination distribution by using the node directions of a geodesic dome. In the proposed method, we assume that light sources in the scene are sufficiently distant from the objects and thus all light sources project parallel rays onto the object surface.

By substituting a collection of imaginary point light sources for the entire illumination, we are able to derive a system of equations in $n$ unknown radiance values of imaginary point light sources from the image irradiance of the shadow image. We then solve for a solution set of unknown radiance values which approximates the illumination distribution of the scene.

The rest of the paper is organized as follows. In Section 2 and Section 3, we explain how to estimate an illumination distribution of a real scene from the image irradiance of a shadow image. We first obtain a formula which relates an illumination distribution of a real scene with the image irradiance of the shadow image (Section 2). Second, by assigning the image irradiance of the shadow image to the formula, we obtain a set of linear equations with unknown illumination radiance values sampled at an equal solid angle. Finally, we solve the set of linear equations for an illumination radiance solution set which represents the illumination distribution of the scene (Section 3). Section 4 shows experimental results of the proposed method applied to real images. To evaluate the accuracy of the illumination distribution estimated by our method, we superimpose a synthetic object with the same shape as that of the occluding object onto an image of the scene. In Section 5, we present concluding remarks.

\section{Formula for Relating Illumination Radi- ance with Image Irradiance}

In this section, we present a formula which relates an illumination distribution of a real scene with the image irradiance of a shadow image. Based on the image formation, the formula is obtained as follows:

1. Illumination radiance to scene irradiance: find a relationship between the illumination distribution of a real scene and the irradiance at a surface point in the scene.

2. Scene irradiance to scene radiance: compute how much of the incoming lights are reflected from the surface toward an image plane.

3. Scene radiance to image irradiance: find a relationship between the reflected light from the surface and the image irradiance at a corresponding point on the image plane.

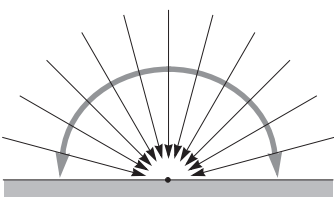

(a)

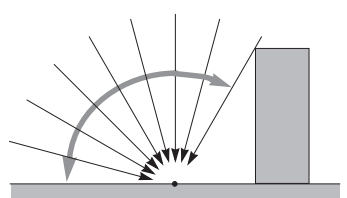

(b)
Figure 1: Total irradiance: (a) without occluding object (b) with occluding object

\subsection{From Illumination Radiance to Scene Irradi- ance}

First, scene irradiance is computed from the entire illumination of the scene. To take illumination from all directions into account, let us consider an infinitesimal patch of the extended light source, of size $\delta \theta_{i}$ in polar angle and $\delta \phi_{i}$ in azimuth as shown in Figure 2.

Seen from the center point $A$, this patch subtends a solid angle $\delta \omega=\sin \theta_{i} \delta \theta_{i} \delta \phi_{i}$. Let $L_{0}\left(\theta_{i}, \phi_{i}\right)$ be the illumination radiance per unit solid angle coming from the direction $\left(\theta_{i}, \phi_{i}\right)$; then the radiance from the patch is $L_{0}\left(\theta_{i}, \phi_{i}\right) \sin \theta_{i} \delta \theta_{i} \delta \phi_{i}[6]$, and the total irradiance of the surface point $A$ is

$$
E=\int_{-\pi}^{\pi} \int_{0}^{\frac{\pi}{2}} L_{0}\left(\theta_{i}, \phi_{i}\right) \cos \theta_{i} \sin \theta_{i} d \theta_{i} d \phi_{i}
$$

Then occlusion of the incoming light by the occluding $o b$ ject is considered as

$$
E=\int_{-\pi}^{\pi} \int_{0}^{\frac{\pi}{2}} L_{0}\left(\theta_{i}, \phi_{i}\right) S\left(\theta_{i}, \phi_{i}\right) \cos \theta_{i} \sin \theta_{i} d \theta_{i} d \phi_{i}
$$

where $S\left(\theta_{i}, \phi_{i}\right)$ are occlusion coefficients; $S\left(\theta_{i}, \phi_{i}\right)=0$ if $L_{0}\left(\theta_{i}, \phi_{i}\right)$ is occluded by the occluding object; Otherwise $S\left(\theta_{i}, \phi_{i}\right)=1$.

\subsection{From Scene Irradiance to Scene Radiance}

Some of the incoming lights at point $A$ are reflected toward the image plane. As a result, point $A$ becomes a secondary light source with scene radiance, which can be computed from scene irradiance at point $A$.

The bidirectional reflectance distribution function (BRDF) $f\left(\theta_{i}, \phi_{i} ; \theta_{e}, \phi_{e}\right)$ is defined as a ratio of the radiance of a surface as viewed from the direction $\left(\theta_{e}, \phi_{e}\right)$ to the irradiance resulting from illumination from the direction $\left(\theta_{i}, \phi_{i}\right)$. Thus, by integrating the product of the BRDF and the illumination radiance over the entire hemisphere, the scene radiance $R s\left(\theta_{e}, \phi_{e}\right)$ viewed from the direction 


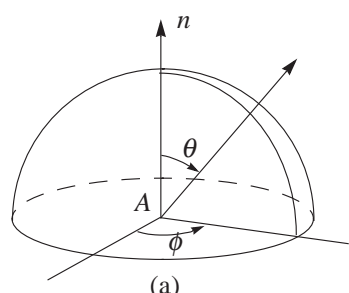

(a)

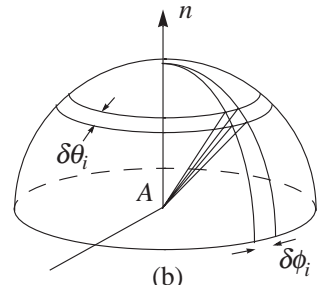

(b)
Figure 2: (a)the direction of incident and emitted light rays (b)infinitesimal patch of an extended light source)

$\left(\theta_{e}, \phi_{e}\right)$ is computed as

$$
\begin{array}{r}
R s\left(\theta_{e}, \phi_{e}\right)=\int_{-\pi}^{\pi} \int_{0}^{\frac{\pi}{2}} f\left(\theta_{i}, \phi_{i} ; \theta_{e}, \phi_{e}\right) L_{0}\left(\theta_{i}, \phi_{i}\right) \\
S\left(\theta_{i}, \phi_{i}\right) \cos \theta_{i} \sin \theta_{i} d \theta_{i} d \phi_{i}
\end{array}
$$

\subsection{From Scene Radiance to Image Irradiance}

Finally, the illumination radiance of the scene is related with image irradiance on the image plane. Since what we actually observe is not image irradiance on the image plane, but rather a recorded pixel value in a shadow image, it is also necessary to consider the conversion of the image irradiance into a pixel value of a corresponding point in the image. This conversion includes several factors such as D/A and $\mathrm{A} / \mathrm{D}$ conversions in a CCD camera and a frame grabber.

Other studies concluded that image irradiance was proportional to scene radiance [6]. In our method, we calibrate a linearity of the CCD camera by using a gray scale chart so that the recorded pixel values also become proportional to the scene radiance of the surface. From Equation 3 the pixel value of the shadow image $P\left(\theta_{e}, \phi_{e}\right)$ is thus computed as

$$
\begin{array}{r}
P\left(\theta_{e}, \phi_{e}\right)=k \int_{-\pi}^{\pi} \int_{0}^{\frac{\pi}{2}} f\left(\theta_{i}, \phi_{i} ; \theta_{e}, \phi_{e}\right) L_{0}\left(\theta_{i}, \phi_{i}\right) \\
S\left(\theta_{i}, \phi_{i}\right) \cos \theta_{i} \sin \theta_{i} d \theta_{i} d \phi_{i}
\end{array}
$$

where $k$ is a scaling factor between scene radiance and a pixel value. Due to the scaling factor $k$, we are able to estimate unknown $L_{0}\left(\theta_{i}, \phi_{i}\right)(i=1,2, \ldots ., n)$ up to scale. To obtain the scale factor $k$, we need to perform photometric calibration between pixel intensity and physical unit $\left(\mathrm{watt} / \mathrm{m}^{2}\right)$ for the irradiance.

\section{Estimation of Illumination Distribution Us- ing Image Irradiance}

After obtaining the formula which relates the illumination radiance of the scene with the pixel values of the shadow image, illumination radiance is estimated based on the recorded pixel values of the shadow image.

\subsection{Approximation of Illuminate Distribution by a Geodesic Dome}

First, the double integral in Equation 4 is approximated by discrete sampling over the entire surface of the extended light source.

Node directions of a geodesic dome are used for approximating the illumination distribution of the scene as a summation of illumination radiance sampled at equal solid angles. Nodes of a geodesic dome are known to be uniformly distributed over the surface of a sphere. Therefore, by using $n$ nodes of a geodesic dome in a northern hemisphere as a sampling direction, the double integral in Equation 4 is approximated as a sampling at an equal solid angle $\delta \omega=2 \pi / n$.

$$
P\left(\theta_{e}, \phi_{e}\right)=\sum_{i=1}^{n} f\left(\theta_{i}, \phi_{i} ; \theta_{e}, \phi_{e}\right) L\left(\theta_{i}, \phi_{i}\right) S\left(\theta_{i}, \phi_{i}\right) \cos \theta_{i}
$$

where $L\left(\theta_{i}, \phi_{i}\right)$ is the illumination radiance per solid angle $\delta \omega=2 \pi / n$ coming from the direction $\left(\theta_{i}, \phi_{i}\right)$, which also includes the scaling factor $k$ between scene radiance and pixel values. The number of the nodes $n$ can be adjusted by changing the sampling frequency of a geodesic dome.

It should be noted that the recorded pixel value $P\left(\theta_{e}, \phi_{e}\right)$ is computed as a function of the illumination radiance $L_{0}\left(\theta_{i}, \phi_{i}\right)$ and the BRDF $f\left(\theta_{i}, \phi_{i} ; \theta_{e}, \phi_{i}\right)$ in Equation 5. We thus take two different approaches depending on whether BRDF of the surface is given in the following sections. We explain the case where the BRDF is given in Section 3.2 and Section 3.3, and the other case where the BRDF is not given in Section 3.4.

\subsection{Known Reflectance Properties: Lambertian Model}

Suppose the surface is a Lambertian surface; BRDF $f\left(\theta_{i}, \phi_{i} ; \theta_{e}, \phi_{e}\right)$ for a Lambertian surface is known to be a constant. From Equation 5, an equation for a Lambertian surface is obtained as

$$
P\left(\theta_{e}, \phi_{e}\right)=\sum_{i=1}^{n} K_{d} L\left(\theta_{i}, \phi_{i}\right) \cos \theta_{i} S\left(\theta_{i}, \phi_{i}\right)
$$

where $K_{d}$ is a diffuse reflection parameter of the surface. From Equation 6, a linear equation is obtained for each image pixel of the shadow image as

$$
a_{1} L_{1}+a_{2} L_{2}+a_{3} L_{3}+\cdots+a_{1 n} L_{n}=P
$$

where $L_{i}(i=1,2, \ldots, n)$ are $n$ unknown illumination radiance specified by $n$ node directions of a geodesic dome. The coefficients $a_{i}(i=1,2, \ldots, n)$ represent $K_{d} \cos \theta_{i} S_{i}$ in Equation 6; we can compute these coefficients from the 3D geometry of a surface point, the occluding object and the 
illuminant direction. ${ }^{1} \quad P$ is the values of the image pixel $P\left(\theta_{e}, \phi_{e}\right)$.

If we select a number of pixels, say $m$ pixels, a set of linear equations is obtained as

$$
\begin{array}{ccl}
a_{11} L_{1}+a_{12} L_{2}+a_{13} L_{3}+ & \cdots+a_{1 n} L_{n} & =P_{1} \\
a_{21} L_{1}+a_{22} L_{2}+a_{23} L_{3}+ & \cdots+a_{2 n} L_{n} & =P_{2} \\
a_{31} L_{1}+a_{32} L_{2}+a_{33} L_{3}+ & \cdots+a_{3 n} L_{n} & =P_{3} \\
& \cdots & \cdots \\
a_{m 1} L_{1}+a_{m 2} L_{2}+a_{m 3} L_{3}+ & \cdots+a_{m n} L_{n} & =P_{m}(8)
\end{array}
$$

Therefore, by selecting a sufficiently large number of image pixels, we are able to solve for a solution set of unknown $L_{i}$ 's [17]. Note that, since each pixel consists of 3 color bands ( $\mathrm{R}, \mathrm{G}$, and $\mathrm{B}$ ), each band of radiance $L_{i}$ is also estimated from the corresponding color band of the image. ${ }^{2}$

\subsection{Known Reflectance Properties: Non-Lambertian Model}

Our method is not limited only to the Lambertian reflection model; but it can also be extended to other reflection models. As shown in the previous case, our method requires a set of linear equations with unknown illumination radiance. Hence, any reflection model is applicable to our method providing such a set of linear equations is obtained. Take a simplified Torrance-Sparrow reflection model $[15,20]$ for example; the pixel value of shadow image $P\left(\theta_{e}, \phi_{e}\right)$ is computed as

$$
\begin{array}{r}
P\left(\theta_{e}, \phi_{e}\right)=\sum_{i=1}^{n}\left(K_{d} \cos \theta_{i}+K_{s} \frac{1}{\cos \theta_{e}} e^{\frac{-\gamma\left(\theta_{i}, \phi_{i}\right)^{2}}{2 \sigma^{2}}}\right) \\
S\left(\theta_{i}, \phi_{i}\right) L\left(\theta_{i}, \phi_{i}\right)
\end{array}
$$

where $\gamma\left(\theta_{i}, \phi_{i}\right)$ is the angle between the surface normal and the bisector of the light source direction and the viewing direction, $K_{d}$ and $K_{s}$ are constants for the diffuse and specular reflection components, and $\sigma$ is the standard deviation of a facet slope of the Torrance-Sparrow reflection model.

From Equation 9, we obtain a linear equation for each image pixel where $L\left(\theta_{i}, \phi_{i}\right)(i=1,2, \ldots, n)$ are unknown illumination radiance, and $\left(K_{d} \cos \theta_{i}+\right.$ $\left.K_{s} \frac{1}{\cos \theta_{e}} e^{\frac{-\gamma\left(\theta_{i}, \phi_{i}\right)^{2}}{2 \sigma^{2}}}\right) S\left(\theta_{i}, \phi_{i}\right)(i=1,2, \ldots, n)$ are known coefficients. Again, if we use a sufficiently large number of pixels for the estimation, we are able to solve for a solution set of unknown illumination radiance $L\left(\theta_{i}, \phi_{i}\right)(i=$ $1,2, \ldots, n)$.

\footnotetext{
1 We established the correspondence between the 3D world coordinate system in the scene and the 2D image coordinate system by using the camera calibration algorithm proposed by Tsai [21]. From the calibration process, a plane of $z=0$ is also defined on the calibration board, onto which the occluding object cast shadows.

2 The question of how to select image pixels to obtain a solution set for unknown radiance values seems to be leading to an interesting research topic. For instance, a similar discussion on this subject can be found in [10].
}

\subsection{Unknown Reflectance Properties : Lamber- tian Model}

Even in the case where the BRDF is not given, we are still able to estimate an illumination distribution of a real scene if the surface is a Lambertian surface. The question we have to consider here is how to cancel the additional unknown number $K_{d}$ in Equation 6.

An additional image of the scene taken without the $o c$ cluding object is used to cancel $K_{d}$. We refer to the image as a surface image. The image irradiance of a surface image represents the surface color of the plane in the case where none of the incoming light is occluded. From this, in the case of the surface image, the shadow coefficients $S\left(\theta_{i}, \phi_{i}\right)$ always become $S\left(\theta_{i}, \phi_{i}\right)=1$. Therefore, using Equation 6, the image irradiance $P^{\prime}\left(\theta_{e}, \phi_{e}\right)$ of the surface image is computed as

$$
P^{\prime}\left(\theta_{e}, \phi_{e}\right)=K_{d} \sum_{j=1}^{n} L\left(\theta_{j}, \phi_{j}\right) \cos \theta_{j}
$$

From Equation 6 and Equation 10, the unknown $K_{d}$ is canceled as

$$
\begin{aligned}
\frac{P\left(\theta_{e}, \phi_{e}\right)}{P^{\prime}\left(\theta_{e}, \phi_{e}\right)} & =\frac{\left.K_{d} \sum_{i=1}^{n} L\left(\theta_{i}, \phi_{i}\right) \cos \theta_{i} S\left(\theta_{i}, \phi_{i}\right)\right)}{K_{d} \sum_{j=1}^{n} L\left(\theta_{j}, \phi_{j}\right) \cos \theta_{j}} \\
& =\sum_{i=1}^{n} \frac{L\left(\theta_{i}, \phi_{i}\right)}{\sum_{j=1}^{n} L\left(\theta_{j}, \phi_{j}\right) \cos \theta_{j}} \cos \theta_{i} S\left(\theta_{i}, \phi_{i}\right)
\end{aligned}
$$

Finally, we obtain a linear equation for each image pixel where $\frac{L\left(\theta_{i}, \phi_{i}\right)}{\sum_{j=1}^{n} L\left(\theta_{j}, \phi_{j}\right) \cos \theta_{j}}(i=1,2, \ldots, n)$ are unknowns, $\cos \theta_{i} S\left(\theta_{i}, \phi_{i}\right)(i=1,2, \ldots, n)$ are computable coefficients, and $\frac{P\left(\theta_{e}, \phi_{e}\right)}{P^{\prime}\left(\theta_{e}, \phi_{e}\right)}$ is a right-hand side quantity. Again, if we use a sufficiently large number of pixels for the estimation, we are able to solve the set of linear equations for a solution set of unknown $\frac{L\left(\theta_{i}, \phi_{i}\right)}{\sum_{j=1}^{n} L\left(\theta_{j}, \phi_{j}\right) \cos \theta_{j}}(i=1,2, \ldots, n)$.

We should point out that the estimated radiance from these equations is a ratio of the illumination radiance in one direction $L\left(\theta_{i}, \phi_{i}\right)$ to scene irradiance at the surface point $\sum_{j=1}^{n} L\left(\theta_{j}, \phi_{j}\right) \cos \theta_{j}$. Hence, without knowing the ratio of the scene irradiance among color bands, there is no way to relate the estimated radiance over the color bands. Our method avoids this problem because of the initial camera calibration. Since we use a white board with regularly spaced dots as a calibration board, the recorded color of the board directly shows the ratio of the scene irradiance among color bands.

\section{Experimental Results}

We have tested the proposed method by using real images of indoor environments. To evaluate the accuracy of the illumination distribution estimated by our method, we 
superimpose a synthetic object with the same shape as that of the occluding object onto an image of the scene taken without the occluding object, and compare the shadows of the synthetic object with those of the occluding object in the shadow image. Section 4.1 explains how to superimpose a synthetic object onto the real scene by using the estimated illumination distribution. In Section 4.2, we describe experimental results in the case where reflectance properties of a reflected surface are known. Then, in Section 4.3, we describe experimental results in the case where reflectance properties of the surface are unknown.

\subsection{Superimposing a Synthetic Occluding Object onto the Scene}

The ray casting algorithm is used to superimpose a synthetic object. If the ray generated from camera projection center through the image pixel intersects a synthetic object, we compute a color to be observed at the surface point using a simplified Torrance-Sparrow reflection model from Section 3.3. From the model, a color to be observed at the surface point $R s\left(\theta_{e}, \phi_{e}\right)$ is computed using the estimated illumination distribution of the real scene as

$$
\begin{aligned}
& R s_{c}\left(\theta_{e}, \phi_{e}\right)=K_{d, c} \sum_{i=1}^{n} L_{c}\left(\theta_{i}, \phi_{i}\right) \cos \theta_{i} \\
&+K_{s, c} \sum_{i=1}^{n} L_{c}\left(\theta_{i}, \phi_{i}\right) \frac{1}{\cos \theta_{e}} e^{\frac{-\gamma\left(\theta_{i}, \phi_{i}\right)^{2}}{2 \sigma^{2}}} \\
& \mathrm{c}=\mathrm{R}, \mathrm{G}, \mathrm{B}
\end{aligned}
$$

where $L_{c}\left(\theta_{i}, \phi_{i}\right)(i=1,2, \ldots, n)$ are the estimated illumination radiance values.

Otherwise, the influence of the synthetic object onto the real object surface is considered. In other words, we create shadows cast by the synthetic object onto the surface.

First, we compute total irradiance $E_{1}$ at the surface point using the estimated illumination distribution in the case where a synthetic object does not occlude any incoming light (Figure 1.a).

$$
E_{1, c}=\sum_{i=1}^{n} L_{c}\left(\theta_{i}, \phi_{i}\right) \cos \theta_{i} \quad c=R, G, B
$$

where $L\left(\theta_{i}, \phi_{i}\right)(i=1,2, \ldots, n)$ are the estimated illumination radiance values.

Second, we compute total irradiance $E_{2}$ at the surface point in the case where the synthetic object occludes some of the incoming light (Figure 1.b).

$$
E_{2, c}=\sum_{i=1}^{n} L_{c}\left(\theta_{i}, \phi_{i}\right) \cos \theta_{i} S\left(\theta_{i}, \phi_{i}\right) \quad c=R, G, B
$$

where $S\left(\theta_{i}, \phi_{i}\right)=0$ if the synthetic object occludes illumination radiance $L\left(\theta_{i}, \phi_{i}\right)$; otherwise, $S\left(\theta_{i}, \phi_{i}\right)=1$.
Then, we compute the ratio of $E_{2}$ to $E_{1}$, which represents how much of the irradiance at the intersection would still be preserved if the synthetic object were placed in the scene. Finally, by multiplying the ratio $E_{2} / E_{1}$ to the observed color of the image pixel $I$, we obtain the color $I^{\prime}$ that would be the color of the image pixel if there were a synthetic object in the scene.

$$
I_{c}^{\prime}=I_{c} \frac{E_{2, c}}{E_{1, c}} \quad c=R, G, B
$$

\subsection{Experimental Results for Known Reflectance Property}

An image of a surface with an occluding object called a shadow image was taken under the usual illumination environment in our office, including direct light sources such as fluorescent lamps and windows to the outside, as well as indirect illumination such as reflections from a wall (Figure 3).

First, an illumination distribution of the scene was estimated using the image irradiance inside shadows in the shadow image as explained in Section 3.2. Then a synthetic object with the same shape as that of the occluding object was superimposed onto an image of the scene taken without the occluding object, called the surface image. Synthesized results are shown in Figure 4 (a), (b), and (c). Also, we superimposed another synthetic object of a different shape onto the scene in Figure 4(d). The number of nodes of a geodesic dome used for the estimation is shown under the resulting image.

We found through our experiments that, the larger number of nodes we used, the more the shadows of the synthetic object resembled those of the occluding object in the shadow image. Especially in the case of 521 nodes, the shadows of the synthetic object are indistinguishable from those of the occluding object in the shadow image: this shows that the estimated illumination distribution gives a good presentation of that of the real scene.

Figure 5 numerically shows the improvement of the accuracy by increasing the number of samplings. The vertical axis represents average error in pixel values inside the shadow regions in the synthesized images compared with those in the shadow image. Here, the initial difference in pixel values of shadow regions between the surface image and the shadow image is set to $100 \%$. The horizontal axis represents the number of nodes of a geodesic dome used for the estimation. From the plot in the figure, we can clearly see that the accuracy improves rapidly as we use more imaginary point light sources.

Also the small pictures right next to the plot shows error distributions inside shadow regions in the synthesized images. Darker color represents larger error in a pixel value in the shadow regions compared with the real shadows of the occluding object in the shadow image. 


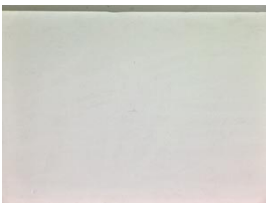

(a)

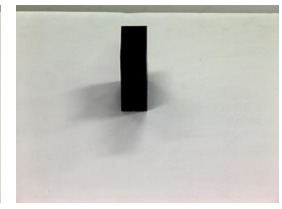

(b)

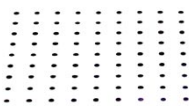

(c)
Figure 3: Input images : (a) surface image (b) shadow image (c) calibration image

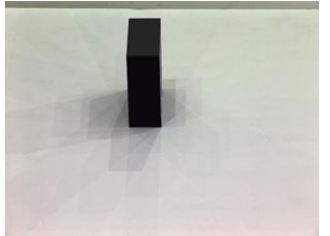

(a) number of nodes : 89

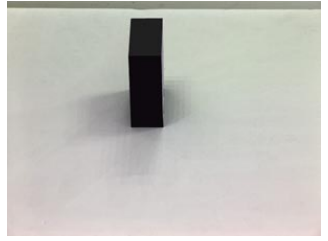

(c) number of nodes : 521

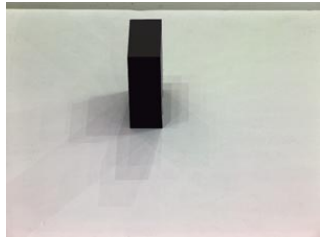

(b) number of nodes : 193

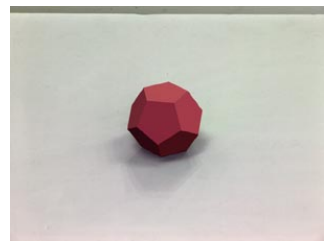

(d) number of nodes : 521
Figure 4: Synthesized images: known reflectance property

Also, the resulting images indicate that it is required to adjust the number of nodes of a geodesic dome depending on the complexity of a scene to obtain a reasonably good estimation for less computational cost. We are currently extending our work so that an appropriate number of nodes is automatically selected for the estimation, depending on the scene complexity.

\subsection{Experimental Results for Unknown Re- flectance Property}

We also applied our method to the case where reflectance properties of a surface were unknown. The input images used in this experiment are shown in Figure 6. Since the reflectance properties of the surface were unknown, the image irradiance of both the shadow image and the surface image were used for estimating the illumination distribution of the scene as explained in Section 3.4.

In the same way as in the previous case, a synthetic occluding object was superimposed onto the surface of the surface image. Synthesized results are shown in Figure 7. Again, in the case of 521 nodes, the shadows in the resulting image strongly resemble those of the occluding object in the shadow image. This shows that the estimated illumination distribution gives a good representation of the characteristics of the real scene.

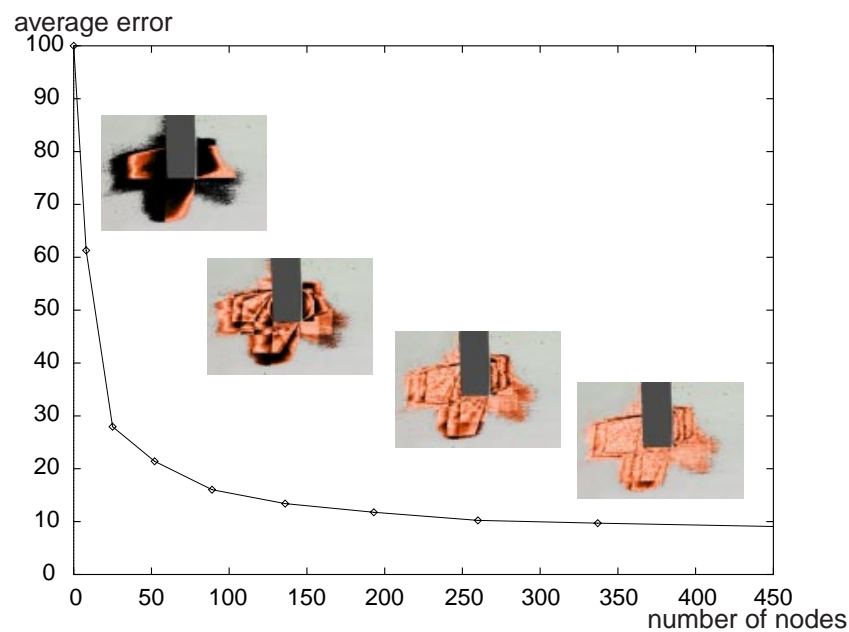

Figure 5: Error Analysis: known reflectance property

We concluded from our experiments that the proposed method is effective for providing an illumination distribution which can be used as a substitution for a real illumination distribution.

\section{Conclusions}

In this paper, we have proposed a new method for estimating an illumination distribution of a real scene from a radiance distribution inside shadows cast by a real object of known shape onto other object surface of known shape and known reflectance. By using the occlusion information of the incoming light, we could estimate an illumination distribution of a real scene reliably even for the images taken in a complex illumination environment.

There have also been several methods proposed for measuring illumination of a real scene in the field of augmented reality research $[3,4,5]$. However, those methods tended to measure the illumination directly from images of the scene and therefore, they suffered from two technical problems: how to capture a wide field of view of the scene, and how to record high dynamic range of the scene. In the proposed method, since we observe shadows and not the illumination itself, no effort to overcome these problems is required.

To demonstrate the effectiveness of the proposed method, we have successfully tested our method by using sets of real images taken in our office with different surface materials of shadow regions.

\section{References}

[1] R. Baribeau, M. Rioux, and G. Godin, "Color Reflectance Modeling Using a Polychromatic Laser Range Sensor" IEEE IEEE Trans. PAMI, vol. 14, no. 2, pp. 263-269, 1992.

[2] J. Bouguet and P. Perona, "3D Photography on Your Desk," Intl. Conference on Computer Vision, pp.43-50, 1998. 


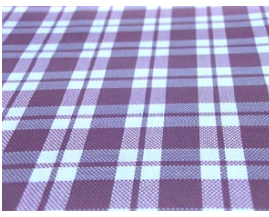

(a)

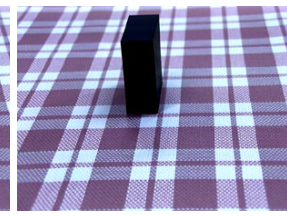

(b)

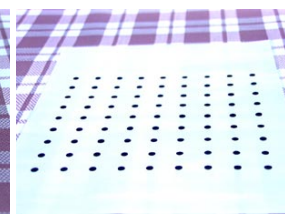

(c)
Figure 6: Input images : (a) surface image (b) shadow image (c) calibration image

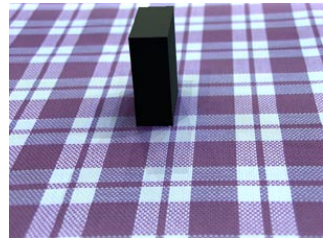

(a) number of nodes : 89

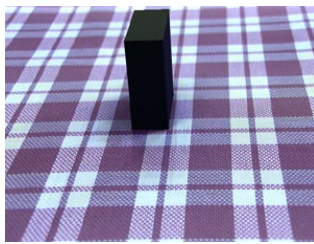

(c) number of nodes : 521
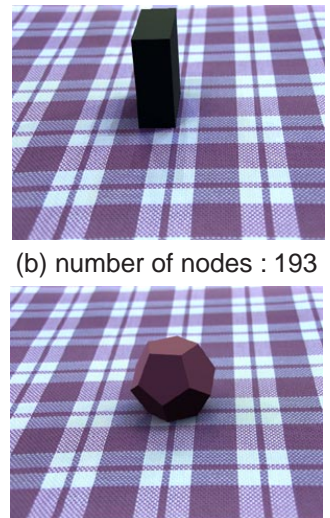

(d) number of nodes : 521 (b) number of nodes : 193

Figure 7: Synthesized images: unknown reflectance property

[3] P. E. Debevec, "Rendering Synthetic Objects into Real Scenes: Bridging Traditional and Image-based Graphics with Global Illumination and High Dynamic Range Photography," Proc. SIGGRAPH 98, pp. 189-198, July, 1998.

[4] G. Drettakis, L. Robert, S. Bougnoux, "Interactive Common Illumination for Computer Augmented Reality" Proc. 8th Eurographics Workshop on Rendering, pp. 45-57, 1997.

[5] A. Fournier, A. Gunawan and C. Romanzin, "Common Illumination between Real and Computer Generated Scenes,"Proc. Graphics Interface '93, pp.254-262, 1993.

[6] B. K. P. Horn, Robot Vision, The MIT Press, Cambridge, MA., 1986.

[7] B. K. P. Horn, "Obtaining Shape from Shading Information," Chapter 4 in The psychlogy of Computer Vision, McGraw-Hill Book Co., New York, N.Y., 1975.

[8] B. K. P. Horn and M. J. Brooks, "The Variational Approach to Shape from Shading," Computer Vision, Graphics, and Image Processing, 33(2), pp.174-208, 1986.

[9] K. Ikeuchi and B. K. P. Horn, "Numerical Shape from Shading and Occluding Boundaries," Artificial Intelligence 17(1-3), pp.141-184, 1981

[10] K. Ikeuchi and T. Kanade, "Automatic Generation of Object Recognition Programs," Proc. IEEE(76), No. 8, pp.1016-1035, 1988

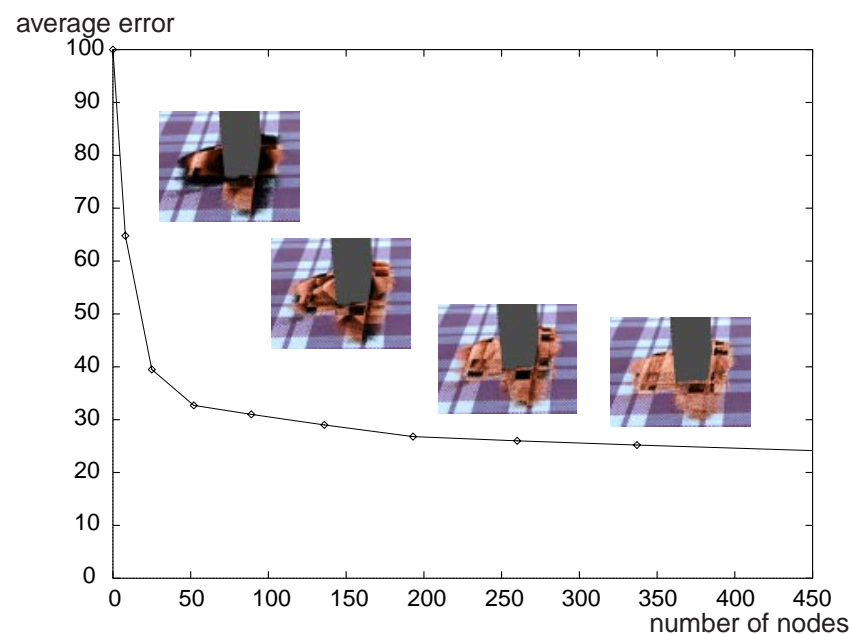

Figure 8: Error Analysis: unknown reflectance property

[11] J. R. Kender and E. M. Smith, "Shape from Darkness: Deriving Surface Information from Dynamic Shadows," Proc. Intl. Conference on Computer Vision, pp.539-546, 1987.

[12] G. Kay and T. Caelli, "Estimating the Parameters of an Illumination Model using Photometric Stereo,"Graphial Models and Image Processing, vol. 57, no. 5, pp. 365-388, 1995.

[13] J. Lu and J. Little, "Reflectance Function Estimation and Shape Recovery from Image Sequence of a Rotating Object,"Proc. IEEE Intl. Conference on Computer Vision '95, pp. 80-86, 1995.

[14] A. K. Markworth, "On the Interpretation of Drawings as ThreeDimensional Scenes," PhD thesis, University of Sussex, 1974.

[15] S. K. Nayar, K. Ikeuchi, and T. Kanade, "Surface reflection: physical and geometrical perspectives," IEEE Trans. PAMI, vol. 13, no. 7, pp. 611-634, 1991.

[16] A. P. Pentland, "Linear Shape From Shading," Intl. J. Computer Vision, 4(2), pp153-162, 1990.

[17] W. H. Press, B. P. Flannery, S. A. Teukolsky, W. T. Vetterling, Numerical Recipes in C: The Art of Scientific Computing, Cambridge University Press, Cambridge, 1988.

[18] Y. Sato, M. D. Wheeler, and K. Ikeuchi, "Object shape and reflectance modeling from observation," Proc. SIGGRAPH 97, pp. 379-387, 1997.

[19] S. A. Shafer and T. Kanade, "Using Shadows in Finding Surface Orientations," Computer Vision, Graphics, and Image Processing, 22(1), pp. 145-176, 1983.

[20] K. E. Torrance and E. M. Sparrow, "Theory for off-specular reflection from roughened surface," J. Optical Society of America, vol.57, pp.1105-1114, 1967.

[21] R. Tsai, "A Versatile Camera Calibration Technique for High Accuracy Machine Vision Metrology Using Off-the-Shelf TV Cameras and Lenses," IEEE J. Robotics and Automation, vol. 3, no. 4, pp. 323-344, 1987. 